\title{
LA ENSEÑANZA DE LA FILOSOFÍA, HOY
}

\author{
Saby Evelyn Lazarte Oyague \\ Universidad Ricardo Palma \\ selo8012@hotmail.com
}

\begin{abstract}
RESUMEN
El presente artículo es un estudio de la situación de la enseñanza de la filosofía en el Perú desde el sistema de la educación nacional y la educación universitaria. Partimos de un análisis de la situación de la filosofía en el siglo XX, para remontarnos a la situación actual a vísperas del bicentenario en el Perú; así desde la experiencia pedagógica y con una visión de análisis observamos la situación de la educación en general y la enseñanza de la filosofía en particular. Por último señalamos la importancia de la filosofía en el siglo XXI, desde una visión humanista, enfatizando que el saber filosófico, provocará un ser humano con mayor sensibilidad social, crítico y dispuesto a la reflexión antropológica, política, ética y moral.
\end{abstract}

\section{PALABRAS CLAVE}

Estadísticas / Educación / Realidad Peruana / Filosofía / Humanidades.

\begin{abstract}
This article is a study of the situation of the teaching of philosophy in Peru since the system of national education and university education. We start with an analysis of the situation of philosophy in the twentieth century, so go back to the current situation on the eve of the bicentennial in Peru; and from the educational experience and a vision of analysis, we look at the situation of education in general and the teaching of philosophy in particular. Finally, we note the importance of philosophy in the twentyfirst century, from a humanistic vision, emphasizing that philosophical knowledge, cause a human being more social, critical and willing to anthropological reflection sensitivity, political, ethical and moral.
\end{abstract}

\section{KEYWORDS}

Statistics / Education / Peruvian Reality / Philosophy / Humanities.

* Ponencia presentada en la Universidad Ricardo Palma, en el ciclo de conferencias por el Día Mundial de la Filosofía, el 14 de noviembre 2012, Auditorio Ricardo Palma. 
La filosofía no es auténtica y fecunda sino en cuanto es reflexión de la realidad (Salazar Bondy, 1969, p.62)

\section{ANTECEDENTES: LA EDUCACIÓN NACIONAL EN LA SOCIE- DAD PERUANA A INICIOS DEL SIGLO XX}

El sistema educativo en el Perú ha tenido innumerables reformas, partiendo del proceso de emancipación después de 1821 observamos que la sociedad se organizó con mayor interés para el desarrollo del país. Desde finales del siglo XIX y sobre todo en el siglo XX el tema de la educación nacional se convierte en tema de discusión de la intelectualidad peruana.

El problema que se abordó es el de la "instrucción nacional", los dirigentes reflexionaron y discutieron sobre la educación y los representantes del Estado organizaron y ordenaron el sistema educativo a partir de las influencias española, francesa y norteamericana, se siguieron modelos educativos ya aplicados en otros países.

Se adoptó un carácter imitativo de los grandes sistemas extranjeros, sin embargo, no se puede negar que fue propio de la época, ver al país como un reflejo de todo lo occidental y europeo se convirtió en un ideal de progreso; pero también una gran parte del país, sobre todo los intelectuales sin representación institucional y política en el Estado, se muestran contrarios al afán imitativo, uno de ellos es José Carlos Mariátegui, quien refiriéndose al proceso de instrucción pública señaló que la educación nacional, en nuestro país, no tiene un espíritu nacional.

En el siglo XX, hasta 1904 el órgano central del movimiento de las ideas pedagógicas fue el Consejo Superior de Instrucción Pública, fundado por imitación francesa en 1876; luego se creó el Ministerio de Instrucción Pública y diferentes planteamientos casi opuestos se impusieron, por un lado la posición de Manuel Vicente Villarán, Joaquín Capelo, Pedro Zulen y por otro la posición de Alejandro Deustua, a pesar de tener entre ellos algunas posiciones encontradas, puesto que, consideraron que uno u otro planteamiento era el mejor, ninguno de estos intelectuales del siglo XX negó a la filosofía como elemento importante de la actividad pedagógica.

Alejandro Deustua en su obra Ensayos Escogidos de filosofía y educación nacional, dice: 
Sólo una pedagogía filosófica, fundada en la teoría de los valores humanos, puede conducir al espíritu directamente hacia los fines sociales que se propone alcanzar la función educadora. Sólo el criterio filosófico puede ofrecer la aptitud de fijar la naturaleza de esos valores, haciendo de la libertad humana al principio fundamental de la educación y de la realización de esos valores. (Deustua, s/f, p. 7).

La importancia de la filosofía y la ciencia para la educación del hombre peruano, se planteó tanto en los discursos inaugurales de los años académicos, así como tambien en los ideales de las políticas públicas planteadas en su momento, Javier Prado en su discurso dirigido a la juventud dice: "se educará a los pueblos para las funciones y deberes que impone la vida nueva, y se orientará firmemente a la juventud, haciendo primar el valor ético sobre el intelectual. Se fortaleceran los caracteres y las energias de las nuevas generaciones y se las educará para la acción, para el trabajo, para la vida y para la patria. La enseñanza será esencialmente realista. Los metodos de observación y experimentación alcanzaran desenvolvimiento ilimitado" (Prado, 1958, p. 38).

\section{LA FILOSOFIA EN LA EDUCACIÓN PERUANA}

En el ambiente educativo nacional, se crean e inauguran los primeros colegios nacionales y facultades, donde la filosofía es considerada como ideal de renovacion nacional. Se busca un mejoramiento de la condicion de vida y un sentido humanista para enfrentar los diversos problemas sociales del país, desde los años 60 en adelante las generaciones se dedicarón a fundamentar filosóficamente los problemas educativos, Walter Peñaloza y Augusto Salazar Bondy se dedicarón a escribir en la historia de la realidad peruana el sentido de humanización del ser humano a partir de los modelos educativos y los planes didáctico pedagógicos; así, según Salazar, la filosofía tiene tres tareas por delante:

a) Una tarea didáctica, de formacion de conciencia: la preparacion racional para la comprensión del mundo y de la existencia de las generaciones más jóvenes;

b) La tarea de afinación de los instrumentos racionales, cuidadoso entrenamiento en las técnicas y los métodos del pensamiento más riguroso, y

c) Una toma de conciencia fundamental de nuestra propia alienación, que debe llevarnos a formar el modelo antropológico de nuestra condición de ser. 
El tema educativo en el pensamiento de Salazar Bondy, está relacionado necesariamente con el planteamiento de los problemas sociales, para que se genere la transformacion total de la colectividad peruana no solamente se debe mirar a la escuela separada de la sociedad, sino que se debe buscar el acoplamiento de la escuela y la sociedad, se debe buscar dice "la educación de la crisis", esto significa hacer una educación que permita levantar la condicion de vida que tiene el país, señala:

(...) no debemos buscar una educación que ignore la situación real del país o que la evite, ni una educación que, por sus propias virtudes y construyendose sin soporte real, pueda salvar al país, sino la educación que nuestra comunidad necesita y sea capaz de construir en estos momentos. Y puesto que el pais vive en crisis, esta educación debe estar marcada por la crisis, debe estar hecha a la medida de las actuales fuerzas nacionales y aprovechando los impulsos y las orientaciones que tratan de sacar al país de su actual situación histórica y cultural. (Salazar, 1969, p. 72)

Nuestra sociedad está en crisis por una falta de reflexión de los hombres que la conforman y la crisis se refleja en las actitudes del hombre y la condicion de vida del hombre, en 1970 Luis Felipe Guerra en su Libro Presentación de la Filosofía dice:

el hombre actual es esencialmente un ser angustiado, que sufre por no saber qué elegir con seguridad, tornándose esto en una situación de permanente inseguridad personal y colectiva, que cada vez va pesando más sobre el destino humano. (Guerra, 1970, p. 18).

A finales del siglo XX el hombre debe ser conciente de la crisis, marcado y educado en esta realidad social, debe disponerse para el progreso y desarrollo, el hombre solo podrá conseguir tan alto ideal, si asume su conciencia de libertad y supera los estados de alienación en el que vive.

Augusto Salazar Bondy, consideró que la filosofía es el pensamiento que permite una reflexión critica, busca formar conciencia por ello es importante educar en filosofía. Apartir del empeño de Salazar se consideró a la filosofía como elemento de acción pedagógica, los planes educativos incluyeron la filosofia en la educación escolar. El planteamiento de Salazar, se articula ahora con la propuesta de metodología de la enseñanza de la filosofía en la educación secundaria, en 1967 se publicó Didáctica de la filosofía, obra donde se muestra la relación directa entre educación y 
filosofía, de las diversas maneras que se puede entender la filosofía lo mas importante es considerar:

a) Una reflexion crítica sobre el conocimiento y la acción,

b) Una concepción del mundo como totalidad,

c) Una orientacion racional, universal de la existencia.

Así a finales del siglo XX la filosofía se incluye y dirige con el afan de concientización del hombre, encontramos entonces una primera razón por la cual la filosofia se torna importante:

la filosofía es, entonces, la última estación de la racionalidad del hombre, en el doble sentido de la más avanzada empresa de la inteligencia y del postrer esfuerzo por comprender el mundo y la vida y por comunicar con los demás. En ella el espiritu humano muestra a la vez su fortaleza y su debilidad. Si la filosofía fracasa, si sus pretenciones de su saber integrador y de máximo rigor no se cumplen, se frustra la aventura del hombre como creador de un orden legal coherente y universal, de un orden en el cual pueden convivir los espiritus. (Salazar Bondy, ¿Qué es filosofia?, 1967, p. 58)

Augusto Salazar Bondy, como filósofo, se preocupó de la importancia de la filosofía para la formación del hombre crítico, del hombre auténtico, y como pedagogo organizó todo un plan articulando un programa para la enseñanza de la filosofía. Junto a él podemos situar a Víctor Li Carrillo (1929-1988), quien se preocupó por la preparación de profesores de filosofía, predispuestos para la enseñanza de la filosofía, algunos de estos jóvenes estudiantes e investigadores, hoy grandes maestras son Martha Zolezzi y Paquita Vexler, ellas son testimonio de la preocupacion por lograr una preparación favorable para la enseñanza de la filosofía.

Víctor Li Carrillo, en su obra La enseñanza de la filosofía, publicado hacia el año 2008, dice:

La enseñanza de la filosofía afronta una situacion, dificil y precaria.

La Evolución del saber, la orientación de la cultura, la organización del mundo actual, parecen incomplatibles no solo con la enseñanza sino con la existencia misma de la filosofía. (Li Carrillo, p. 37)

En las últimas tres decadas del siglo $\mathrm{XX}$, la preocupación de Walter Peñaloza, Augusto Salazar Bondy y Víctor Li Carrillo, provocó 
la incorporación de la filosofía como un curso básico para la formación escolar, se consiguió finalmente que la filosofía pueda forjar hombres libres, reflexivos y críticos, que puedan responder a los problemas de la "crisis". Sin embargo, los sueños no duraron mucho, tras la década del noventa, las nuevas políticas educativas del sistema, aniquilaron y suprimieron el "curso de filosofía" de las escuelas y con ello la importancia de formar conciencia.

\section{LA FILOSOFIA EN LA EDUCACIÓN NACIONAL DEL SIGLO XXI}

El siglo XXI, se nos presenta como el siglo del desarrollo económico, del progreso social, de la globalización y la cibernética, pero sin filosofía; "filosofía”, “para qué?”, muchos señalan que estamos en la época de los negocios y asuntos prácticos. Después de la década del noventa, ya son doce años que la filosofía no es considerada como un curso del sistema educativo nacional.

El sistema educativo nacional, articulado en tres modalidades, Educación Básica Regular (EBR), Educación Básica Alternativa (EBA) y Educación Básica Especial (EBE), aplican un Diseño Curricular Nacional, conocido como (DCN), donde se encuentra la política nacional en cuanto a educación, inicial, primaria y secundaria de todo el pais, se aplica en el Perú bajo un esquema de áreas curriculares. EL DCN presenta actualmente dos documentos es decir; uno para la EBR, EBE; y otro para la EBA. Ambos documentos presentan sus componentes y características, los componentes en EBR son los objetivos, contenidos, metodología y evaluación; en EBA está referido a los aprendizajes a lograr y las competencias. Las características del DCN son cinco 1) Básico, 2) Humanista y valorativo, 3) Conceptualmente abierto, 4) Integral y 5) Interdisciplinario, y las características en EBR son tres 1) diversificable, 2) abierto y 3) flexible. La organización de la (EBR) es a modo de niveles, ciclos, grados yen (EBA) ciclos, programas y formas de atención. Las áreas curriculares están organizadas para EBR y EBA por campos temáticos de estudio, sin embargo en ninguno de los dos diseños curriculares se observa explictamente el curso de filosofía.

\section{LA FILOSOFIA EN LA UNIVERSIDAD DEL SIGLO XXI}

En el sistema universitario, la filosofía se mantuvo, como carrera profesional en la antiquisima Universidad Nacional Mayor de San Marcos; 
tras la supresión de la filosofía paradojicamente para el año 2000 se creaba la escuela de filosofía en la Universidad Nacional Federico Villarreal, tambien se mantiene la filosofía en la Universidad Católica del Perú, Universidad Pontificia y Civil de Lima y La Universidad Antonio Ruiz de Montoya.

En otras universidades, aún se conserva la filosofía, como una especialidad de la carrera de educación como es el caso de la Universidad Enrique Guzmán y Valle (La Cantuta) y tambien en Universidades de otras ciudades del Perú como son Arequipa, Cuzco, Ayacucho, Iquitos, Trujillo, Huánuco entre otras.

En universidades con otras carreras como Medicina, Ingeniería, Arquitectura, Administración, carreras que al parecer nada tienen que ver con filosofía. El sistema universitario hoy valora y considera la filosofía como en la Universidad Ricardo Palma, que por medio del Programa de Estudios Básicos, se fortalece la visión humanística con el curso de filosofía. Con el lema "Formamos seres humanos para una cultura de paz", la universidad con tendencia humanista fortalece la razón de ser de la filosofía, razón que nos permite celebrar cada tercer jueves de noviembre el día mundial de la filosofía.

\section{SIN FILOSOFÍA EN EL SIGLO XXI}

Las consecuencias tras la supresion de la filosofia, entre otros son:

1) Un bajo rendimiento académico en el razonamiento matemático y verbal.

2) Un total desapego por la lectura.

3) Sujetos alienados, bajo los parametros del capitalismo y la sociedad del consumo.

4) Personas superficiales, frias y sin valores.

5) Sujetos sin ideas propias, y con casi nada de reflexión y espíritu crítico

\section{LA IMPORTANCIA DE LA ENSEÑANZA DE LA FILOSOFÍA}

Consideramos que la enseñanza de la filosofia en el Perú del siglo XXI, es importante en tanto que se provoque cambios en al menos seis áreas importantes del ser humano, del peruano de hoy. La filosofía es importante: 
1) Para la individuo, porque fortalece el ser humano responsable y libre.

2) Para la familia, porque procura un sentido de formación moral con valores.

3) Para el profesional, porque induce a una conducta etica.

4) Para la empresa, porque dirige su mirada hacia la condición humana.

5) Para la sociedad, porque permite el respeto del "otro" con dignidad.

6) Para el Estado, porque genera reflexión y crítica en las políticas públicas.

La filosofía provoca en el ser humano que asuma su existencia con dignidad, siendo auténticos, solidarios y reflexivos y esto va de la mano con la enseñanza de la filosofía lo cual bosqueja la educación que queremos para la generación del Bicentenario del Perú (continuará).

\section{REFERENCIAS BIBLIOGRÁFICAS}

DEUSTUA, Alejandro

s/f Ensayos escogidos de filosofía y educación nacional. Lima: Primer festival del libro de Junin.

GUERRA, L. F.

1970 Presentacion de la Filosofía. Lima: Casa de la Cultura del Perú.

LI CARRILLO, Víctor

2008 La enseñanza de la filosofía. Lima: Fondo editorial UIGV.

PRADO, Javier

1958 Discurso del maestro de la juventud. En B. d. Peruano, Palabras a la juventud (págs. 29-48). Lima: Ediciones del Ministerio de Educación Pública.

SALAZAR BONDY, Augusto

1967 ¿Qué es filosofia? Lima: s/e.

1969 Entre Escila y Caribdis. Reflexiones sobre la vida peruana. Lima: Casa de la cultura del Perú. 